\title{
LINGUISTIQUE
}

\author{
MAŁGORZATA BALICKA
}

Università Adam Mickiewicz, Poznań

\section{ITALIANO, FRANCESE, POLACCO: NEL TRIANGOLO DELLE BERMUDA DELL'INTERFERENZA LINGUISTICA}

\begin{abstract}
Balicka Matgorzata, Italiano, francese, polacco: nel Triangolo delle Bermuda dell interferenza linguistica [Italian, French, Polish: in the Bermuda Triangle of linguistic interference]. Studia Romanica Posnaniensia, Adam Mickiewicz University Press, Poznań, vol. XXXVI: 2009, pp. 3-14. ISBN 978-83-232-2035-0. ISSN 0137-2475.

Increasing contacts between nations make people learn foreign languages. Unfortunately, the use of more than one language system may cause errors due to linguistic interference. In the present paper we consider different definitions of this phenomenon and describe the conditions required for its presence, along with proposals on how to eliminate interference mistakes. A specific case is false friends, words similar in form but different in meaning, which can lead to misunderstandings or even disgrace. As interference implies the knowledge of at least two languages, bilingualism is another problem discussed in the paper. We present different approaches to interference and study the relation between bilingualism and interference. Finally, we discuss loanwords as one of the results of interference. With all these considerations in mind we can make a specific study of interference mistakes made by Polish students of Italian philology.
\end{abstract}

Nel mondo di oggi la conoscenza delle lingue straniere sembra indispensabile. Tuttavia, usando più di un sistema linguistico si corre il rischio di mescolare sistemi oppure di creare delle forme ibride. Nel presente articolo analizzeremo il fenomeno dell'interferenza percepito in modo diverso da vari studiosi e le condizioni dell'apparizione dell'interferenza linguistica, indicheremo inoltre le soluzioni per ridurre il numero di errori interferenziali. Un caso particolarmente interessante sono $i$ falsi amici, espressioni in due lingue affini quanto alla forma ma diverse quanto al significato. Siccome l'interferenza suppone la conoscenza di più lingue, un altro termine da prendere in considerazione è il bilinguismo. Analizzeremo varie definizioni del termine bilinguismo proposte da linguisti e la relazione tra il bilinguismo 
e l'interferenza. Alla fine ci occuperemo dei prestiti che possono scaturire dagli errori interferenziali. Dopo aver approfondito i fenomeni sopracitati presenteremo gli errori di studenti di filologia italiana commessi nell'espressione scritta e parlata in italiano ed in francese.

\section{FENOMENO DI INTERFERENZA}

Una delle definizioni dell'interferenza linguistica considera il fenomeno come «la deviazione dalla norma che appare nel parlare delle persone bilingue dovuta alla conoscenza di più di una lingua» ${ }^{1}$ (Weinreich, 1953: 1). La presente definizione dà la visione globale e costituisce la base per gli studi successivi del problema. L'errore viene percepito come una deviazione, un elemento erroneo da eliminare che ostacola la comprensione e il buon uso della lingua. Siccome l'interferenza può accadere in vari sottosistemi linguistici Weinreich individua tre tipi di interferenza (Weinreich, 1953: 14-62):

1) interferenza fonica,

2) interferenza grammaticale,

3) interferenza lessicale.

Nel caso dell' interferenza fonica vengono distinti i meccanismi seguenti:

a) sotto-differenziazione di fonemi (under-differentiation of phonems) consiste nel non distinguere $\mathbf{i}$ due fonemi della lingua seconda quando nella lingua prima esiste un solo fonema

b) sopra-differenziazione di fonemi (over-differentiation of phonems) è un errore che consiste nel fare una distinzione di fonemi inesistente nella lingua seconda sotto l'influsso della lingua prima in cui la distinzione del genere è presente,

c) reinterpretazione delle distinzioni (reinterpretation of distinctions) accade quando si distinguono dei fonemi del sistema secondario attraverso tratti pertinenti nel sistema primario ma poco rilevanti nel sistema secondario,

d) sostituzione di fonemi (phone substitution) succede quando la descrizione dei fonemi nelle due lingue è la stessa ma la loro realizzazione fonica è diversa. del fenomeno:

Per quanto riguarda l'interferenza grammaticale, Weinreich individua tre tipi

a) uso dei morfemi del sistema primario nel sistema secondario,

b) applicazione delle regole grammaticali del sistema primario nel sistema secondario oppure negligenza delle regole del sistema secondario inesistenti nel sistema primario,

c) cambiamento (estensione oppure riduzione) della funzione del morfema del sistema secondario secondo il modello del sistema primario.

\footnotetext{
1 " those instances of deviation from the norms of either language which occur in the speech of bilinguals as a result of their familiarity with more than one language $[. .$.$] will be referred to as inter-$ ference phenomena "
} 
Nel caso dell'interferenza lessicale lo studioso distingue:

a) transfert degli elementi lessicali dalla lingua prima alla lingua seconda,

b) estensione dell'uso di una parola del sistema secondario secondo l'uso del suo equivalente nella lingua prima.

Le distinzioni presentate permettono di percepire il problema in modo dettagliato e rendono il carattere complesso del fenomeno. Con la sua tipologia Weinreich inizia gli studi sull'interferenza ripresi successivamente da vari linguisti che presentano la loro definizione del problema. Uno dei linguisti polacchi, L. Grochowski definisce l'interferenza come « l'azione rallentante e deformante delle abitudini linguistiche della lingua materna sulle abitudini linguistiche della lingua seconda, il cui risultato è la deviazione dalle norme della lingua seconda in ogni suo sistema ${ }^{2}$ (Grochowski, 1979: 567). Grochowski si basa sulla definizione weinreichiana sottolineando l'effetto negativo dell'interferenza e ammettendo solo la direzione dalla lingua prima alla lingua seconda. Un'opinione diversa viene espressa da J.A. Czochralski: l'interferenza è " l'influsso reciproco delle strutture appartenenti a due diversi sistemi linguistici " ${ }^{3}$ (Czochralski, 1979: 521). Come si può osservare, non viene menzionata la direzione in cui avviene l'interferenza né lo status dei sistemi linguistici (se sono la lingua prima e la lingua seconda oppure due lingue seconde come nel caso degli studenti polacchi di filologia italiana). Lo stesso linguista individua l'interferenza positiva, l'uso sbagliato delle strutture e l'interferenza negativa che consiste nell'evitare gli elementi di un sistema inesistenti nell'altro sistema in questione.

Accanto al termine interferenza in varie pubblicazioni appare il termine transfert di provenienza psicologica che significa " l'uso automatico, non controllato e inconscio dei comportamenti appresi in precedenza durante la creazione di comportamenti nuovi ${ }^{4}$ (Lipińska, 2003: 80). Sul campo linguistico il transfert riguarda l'uso automatico delle strutture apprese in precedenza in contesti nuovi.

Lo stesso termine viene impiegato da $\mathrm{H}$. Komorowska che individua il transfert positivo, negativo e zero (inesistente). Secondo la linguista polacca, il transfert positivo è l'influsso che facilita l'apprendimento delle nuove capacità mentre il transfert nagativo lo ritarda. Oltre a questa tipologia, Komorowska individua due tipi di transfert negativo: il rallentamento proattivo, quando una capacità precedente influenza una capacità successiva e il rallentamento retroattivo, quando una capacità successiva influenza una capacità precedente.

${ }^{2} \ll[\ldots]$ interferencja jest to hamujące [...] i zniekształcające [...] działanie ojczystych nawyków mownych na kształtowane nawyki mowne $w$ języku obcym, którego rezultat stanowi odchylenie od norm danego języka obcego we wszystkich jego systemach ».

${ }^{3}$ « wzajemne oddziaływanie na siebie struktur językowych należących do dwóch różnych systemów językowych nazywamy interferencją językową".

${ }^{4}$ « [...] termin ,transfer" pochodzi z psychologii i oznacza automatyczne, niekontrolowane i nieświadome używanie wcześniej wyuczonych zachowań przy próbie wytworzenia nowych ». 
Terence Odlin (Odlin, 1989: 36-38) sottolinea la differenza tra l'interferenza (di carattere negativo) e il transfert (di carattere sia positivo che negativo), l'influsso di una lingua su un'altra che facilita oppure ostacola l'apprendimento. Nell'ambito del transfert negativo lo studioso americano individua:

a) sottoproduzione, quando si evitano le strutture della lingua seconda inesistenti oppure molto diverse dalle strutture nella lingua prima,

b) sovraproduzione, quando si usano le strutture in modo eccessivo, può essere il risultato della sottoproduzione se le strutture omesse vengono sostituite da altre oppure quando una struttura è più frequente in una delle lingue parlate,

c) errori di produzione tra cui sostituzioni (uso delle forme della lingua prima nella lingua seconda), calchi (traduzioni letterarie delle struture fisse) e alternazioni di strutture (per esempio sotto la forma di ipercorrezioni).

L'ultima classificazione da noi presentata, di cui autore è Aleksander Szulc, assomiglia a quella proposta da Weinreich anche se si notano certe differenze. Szulc individua vari tipi di interferenza in rapporto al piano linguistico in cui essa opera:

a) interferenza sul piano di espressione di tipo segmentale e soprasegmentale (con successive sottocategorie),

b) interferenza sul piano semantico di tipo grammaticale (formale, morfologica e sintattica) e lessicale,

c) interferenza sul piano pragmalinguistico.

Conoscendo il carattere del fenomeno si devono stabilire le condizioni in cui esso appare più spesso. Szulc ritiene che l'influsso di una lingua su un'altra dipenda dal grado di chiusura del sottosistema; il sottosistema più esposto all'interferenza è quello lessicale che offre la maggiore arbitrarietà nella scelta dei lessemi (Szulc, 1979: 633-635). Questa regola non è sempre valida: sul piano di espressione il sottosistema fonemico è quello più determinato e si potrebbe credere che non sia toccato dall'interferenza, invece il parlante sostituisce gli allofoni della lingua seconda con quelli della lingua prima ed è proprio la pronuncia a rivelare che l'interlocutore è straniero.

Un'altra questione interessante è la direzione in cui avviene l'interferenza. Di solito il sistema primario influenza il sistema secondario ma nel caso della perfetta padronanza della lingua seconda essa può influire sulla lingua prima. Se uno conosce più lingue, due sistemi secondari possono influire uno sull'altro come nel caso degli studenti di filologia italiana che commettono errori di interferenza tra italiano e francese, di cui nessuno è la loro lingua primaria. Inoltre, Czochralski osserva che la struttura più forte (quella imparata meglio e impiegata più frequentemente) influenza quella più debole ma la forza di un sistema e la debolezza dell'altro possono avere il carattere momentaneo (Czochralski, 1979: 540-541).

Un altro problema è il grado in cui la distanza tra i due sistemi determina l'interferenza. Komorowska sostiene che il numero di errori interferenziali aumenta quando le strutture delle due lingue sono simili ma non identiche (Komorowska, 1980: 116). Quell'opinione non viene condivisa da alcuni studiosi. Secondo 
H.V. George la corrispondenza delle due lingue facilita l'apprendimento (George, 1972: 161) mentre W.M. Rivers sostiene che la possibilità dell'interferenza è la più alta quando le due lingue divergono di più (Rivers, 1972: 17).

L'ultima questione ma probabilmente quella più importante è come prevenire l'interferenza. Komorowska propone le ripetizioni molteplici, il confronto delle due forme che provocano errori e il collegare delle strutture ai diversi elementi situazionale in cui esse vengono usate (Komorowska, 1980: 120-128). Una strategia più complessa viene proposta da A. Prejbisz secondo il quale la prevenzione dell' interferenza consiste nel contatto con la cultura del dato paese straniero e nella scelta adeguata dei materiali didattici (Prejbisz, 1964: 217), l'opinione divisa da vari altri linguisti. Oltre alle idee menzionate sopra bisogna citare la soluzione proposta da F. Grucza che suggerisce la separazione dei ruoli linguistici dei genitori. Rivolgendosi al bambino, la madre e il padre parlano ognuno la propria lingua materna per non mescolare i due sistemi linguistici (Grucza, 1981: 34).

Il caso particolare dell'interferenza linguistica sono i falsi amici, simili in forma ma diversi in significato. La loro apparente somiglianza diventa la causa dell'incomprensione, anzi del discredito. In questo punto si deve accennare al fatto che a volte $\mathrm{i}$ falsi amici hanno i significati simili (ma non identici) grazie all'etimologia comune; ci sono anche dei casi in cui una parola polisemica in una lingua ha l'equivalente nell'altra lingua ma solo nell'ambito di un significato. Un'altra difficoltà è la connotazione delle parole. Spesso in una lingua la parola è neutra mentre nell'altra ha il carattere decisamente positivo o negativo. A titolo di esempio citiamo la coppia di parole aglomeracja e agglomeration. Il termine polacco è neutro denominando un insieme degli edifici e persone sulla superficie determinata mentre il termine inglese esprime l'idea del raggruppamento disordinato di elementi diversi (Rudolf, 2003: 102).

Oltre alle differenze semantiche esiste il problema delle divergenze grammaticali come la differenza del genere (la mer ma il mare) oppure la mancanza dell' equivalente in una data categoria grammaticale. Per esempio in polacco esiste un sostantivo, un aggettivo ed un avverbio: efekt, efektowny, efektownie mentre la sola forma francese è effet, mancano l'aggettivo e l'avverbio (Wilczyńska, Rabiller, 1995: 7, 73).

Dalle nostre considerazioni risulta che il primo passo verso l'eliminazione degli errori è proprio la consapevolezza che essi esistono.

\section{BILINGUISMO - DEFINIZIONI}

Nell'opinione comune è bilingue l'individuo che parla due lingue in modo perfetto come se fossero due lingue materne, di solito in seguito alla provenienza dalla famiglia mista. In altri casi il fenomeno non viene percepito in modo univoco. Vari linguisti definiscono il bilinguismo in modo diverso, esso può essere « l'uso 
a turno di due lingue $»^{5}$ (Weinreich, 1953: 1); per altri ogni individuo che impara una lingua straniera diventa bilingue in un certo grado (Szulc, 1979: 632) oppure è la capacità di pensare nella data lingua straniera a decidere della sua perfetta padronanza (Grochowski, 1979: 558). Il bilinguismo viene anche definito come «la padronanza di due lingue in grado simile alla padronanza della lingua materna ${ }^{6}$ nell'idea bloomfieldiana citata da Woźniakowski (Woźniakowski, 1981: 41). Altri studiosi aggiungono certi criteri del bilinguismo: le due lingue devono svolgere regolarmente il ruolo del mezzo di comunicazione e si deve essere capaci di produrre degli enunciati completi e coerenti nella seconda lingua. Siccome le definizioni presentate sono imprecise si suggerisce l'analisi del fenomeno secondo cinque criteri: grado di bilinguismo, grado di diversificazione tra le lingue, età in cui viene imparata la lingua seconda, modo di apprendimento e atteggiamento verso la lingua seconda. In ogni categoria si individuano vari tipi di bilinguismo (Woźniakowski, 1981: 42-43).

Una tipologia simile appare nell'articolo di Grucza che individua i criteri seguenti: limite del bilinguismo (individuale o sociale), età e condizioni di apprendimento, grado di padronanza, status delle lingue, funzionamento comunicativo delle lingue e rapporto tra bilinguismo e acculturazione (Grucza, 1981: 10-11).

La questione del grado di padronanza della lingua che permette di considerare un individuo bilingue appare negli articoli di vari linguisti citati nell'articolo di K. Wróblewska-Pawlak. A. Martinet ritiene che si diventa bilingui quando si è capiti in una lingua diversa dalla propria lingua materna, J. Macnamara sostiene che la condizione minima del bilinguismo stia nell'avere almeno una delle competenze linguistiche della lingua seconda mentre F. Grosjean spiega il bilinguismo come "l'uso regolare delle due lingue " (Wróblewska-Pawlak, 2003: 455-456). Wróblewska-Pawlak ha eseguito un'inchiesta tra gli studenti di filologia romanza chiedendogli se si considerassero bilingui oppure no, e perché. Gli studenti che si consideravano bilingui hanno motivato la risposta con la capacità di comunicare fluentemente, di esprimere propri pensieri e di tradurre senza preparazione. Comunque, alcuni di loro hanno ammesso di non conoscere in grado sufficiente la mentalità e la cultura dei francesi. Gli studenti che non si consideravano bilingui hanno giustificato la loro opinione con la presenza di errori nella produzione in francese, con il fatto di non pensare in questa lingua e con l'ignoranza di linguaggi settoriali. C'erano anche gli studenti convinti che per essere bilingue si deve imparare la lingua sin dall'infanzia.

La divergenza tra l'opinione comune e la definizione scientifica è palese: si tende a descrivere il bilinguismo come la conoscenza perfetta di due lingue mentre la teoria di Weinreich che lo considera come l'uso alternativo di due lingue lascia più possibilità di interpretazione. C. Bettoni avanza la proposta di ordinare il bilinguismo in varie categorie e di delineare i suoi limiti (Bettoni, 1999: 230-234).

\footnotetext{
5 « the practice of alternately using two languages will be called bilingualism ».

6 « opanowanie dwu języków w stopniu podobnym do języka ojczystego ».

" regularne używanie dwóch języków ».
} 
Un'altra questione da prendere in considerazione è il valore del fenomeno. Nel mondo odierno si tende a trattare il bilinguismo come un fenomeno positivo ma esistono tesi che accusano il bilinguismo di svolgere l'influsso negativo sull'intelligenza del bambino. In seguito si è creduto che il bilinguismo non controllato conducesse al miscuglio di lingue. Comunque, gli studi più recenti hanno rilevato che bambini bilingui superano quelli monolingui nei test linguistici.

Di solito la lingua materna influenza la lingua straniera; nel caso di persone plurilingui il problema è più complesso. L'interferenza avviene non solo tra il sistema primario e quello secondario ma anche tra due sistemi secondari. Come prevedere l'apparizione di errori interferenziali? Secondo la regola, il sistema dominante influenza quello più debole, allora bisogna determinare la posizione della data lingua conosciuta dall'individuo plurilingue. Weinreich propone sette criteri che permettono di individuare il sistema più forte:

1) abilità linguistica relativa - la migliore conoscenza della lingua le dà la posizione dominante,

2) rafforzamento visuale - la lingua in cui si scrive e si legge diventa quella dominante,

3) ordine ed età di apprendimento - di solito la lingua imparata come prima è la più forte,

4) utilità comunicazionale - il sistema usato più spesso diventa quello dominante,

5) coinvolgimento emozionale - si crea l'affetto per la lingua in cui si impara a nominare la realtà, di solito è la lingua materna,

6) funzione nel passaggio alla classe sociale superiore - il sistema che decide dell'ascesa nella gierarchia sociale può diventare il più importante

7) valore letterario - culturale - la lingua dalla tradizione artistica diventa quella dominante.

Ovviamente, diversi criteri possono nominare varie lingue come quelle dominanti (Weinreich, 1953: 74-80). Inoltre, Weinreich sostiene che l'individuo bilingue, parlando con un monolingue, tende a limitare l'interferenza esprimendosi solo nella lingua conosciuta dall'interlocutore. Quando invece la conversazione si svolge tra due bilingui che conoscono le stesse lingue loro tendono a mescolare i sistemi, allora l'interferenza non viene limitata in nessun modo. Bisogna anche notare che spesso i bilingui sono capaci di discutere certe questioni solo in una delle lingue e la necessità di trattarle nell'altro sistema provoca subito errori di interferenza.

\section{INTERFERENZA E PRESTITO}

Analizzando il fenomeno di interferenza dobbiamo rispondere alla domanda in quale misura l'interferenza sia diversa dal prestito. Ambedue i processi introducono degli elementi nuovi nella lingua ma nei particolari essi differiscono in modo decisivo. La prima differenza sta nel fatto che nel caso del prestito gli elementi 
stranieri vengono introdotti nella lingua materna, mentre nel caso dell'interferenza il senso dell'influsso è opposto (dalla lingua materna verso la lingua straniera). In seguito, i prestiti arricchiscono una lingua mentre l'interferenza può essere fonte di errori. Inoltre, il prestito è un processo consapevole e arbitrario mentre l'interferenza è di solito inconsapevole (tranne alcune eccezioni). Infine, il prestito ha il carattere collettivo, è un fenomeno sociale dato che un'espressione nuova viene utilizzata dall'intera comunità linguistica mentre gli errori interferenziali capitano negli atti comunicativi individuali e non sono mai accettati dalla comunità per la quale una data lingua è la lingua materna. In questo modo il prestito funziona sul livello della langue mentre l'interferenza sul livello della parole. Si deve anche sottolineare che il prestito è un processo diacronico dai risultati duraturi mentre l'interferenza è un processo sincronico i cui risultati non influenzano in nessun modo il sistema in cui essa opera.

Tuttavia, l'interferenza può essere il punto di partenza per il prestito quando molti membri della comunità linguistica commettono gli stessi errori interferenziali sotto l'influsso di un altro sistema linguistico. Lo stesso Weinreich sostiene che i fenomeni interferenziali ripetuti dai bilingui finiscono con stabilirsi nella lingua e il loro uso non dipende più dal bilinguismo (Weinreich, 1953: 11). In certi casi una parola straniera ha sostituito la parola originaria in modo che tutta la comunità, anche gli individui monolingui, la usino senza consapevolezza del suo carattere di prestito. Gusmani spiega il fenomeno nel modo seguente: il prestito appare nell'atto linguistico individuale per poi diffondersi tra altri parlanti. L'innovatore si ispira al modello straniero riproducendo nella propria lingua il modello tratto da un'altra lingua. Contrariamente a certe opinioni, Gusmani sostiene che un prestito entrato nella lingua non si differenzi più da altre strutture della lingua (Gusmani, 1981: 11-12).

\section{PRESTITI - TIPOLOGIE}

Come nel caso di altri fenomeni, anche nel campo dei prestiti vengono proposte varie tipologie di cui ne citiamo quella di Edward Łuczyński e Jolanta Maćkiewicz. A seconda dell'oggetto del prestito si individuano i prestiti propri quando si prendono in prestito le parole assieme ai loro significati (p.es. computer) e i prestiti artificiali nel caso di prendere dei morfemi stranieri di una o più lingue e poi costruire da essi una parola secondo le regole morfologiche di una data lingua (p.es. televisione dal greco tele - a distanza e dal latino visio - il vedere). Accanto a questi esistono anche i prestiti strutturali (chiamati anche calchi lessicali) in cui le parole straniere vengono tradotte morfema dopo morfema. Per esempio il tedesco Zeitschrift ("tempo + scrittura" dal senso 'rivista') è stato tradotto in polacco come $c z a$ sopismo (czas - tempo e pismo - scrittura). Si possono tradurre anche delle strutture intere - in questo caso parliamo dei calchi fraseologici come il polacco ostatni 
krzyk mody e l'italiano ultimo grido della moda, traduzioni esatte del francese dernier cri de la mode. Gli ultimi nel presente elenco sono i prestiti semantici che consistono nell'allargare il campo semantico di una parola secondo il modello di una parola straniera polisemica.

Secondo il grado dell'assimilazione si individuano le citazioni che entrano nella lingua nella loro forma originale (come il francese menu). Le citazioni conservano la loro forma fonica e grafica straniera e non si sottopongono alla flessione né alla derivazione. In seguito, esistono dei prestiti assimilati parzialmente. In questa categoria possiamo individuare certi gradi di assimilazione. Il primo gruppo viene costituito dai prestiti dalla forma originale che si sottopongono sia alla flessione sia alla derivazione (p.es. pizza che in polacco si sottopone alla declinazione ma meno alla derivazione, anche se si cerca di creare neologismi tipo pizzernia [luogo dove si serve la pizza] che però non sono tanto diffusi e non appartengono alla lingua ufficiale). Il secondo gruppo sono i prestiti dalla forma originale che si sottopongono ai due processi (come weekend). Il terzo gruppo è costituito dai prestiti adattati in modo grafico, fonetico, flessivo e derivativo (come komputer scritto alla polacca). L'ultimo gruppo dei prestiti sono i prestiti interamente assimilati in cui accanto agli adattamenti menzionati incontriamo dei cambiamenti morfologici come l'aggiunta della desinenza tipica della lingua di arrivo (p.es. szarlotka con la desinenza polacca dal francese charlotte) (Euczyński, Maćkiewicz, 1999: 92-93).

Alla fine analizziamo i motivi dell'apparizione dei prestiti. La prima causa è la ovvia necessità di nominare dei concetti nuovi. Un altro motivo è la frequenza bassa di una parola che può essere facilmente sostituita da un'altra, più stabile. Anche la somiglianza alle parole già esistenti, p.es. l'omonimia, può favorire l'introduzione delle voci straniere. Come la spinta all'accoglimento di prestiti si cita anche il bisogno di sinonimi, soprattutto per le parole poco « eleganti $»^{8}$. Un altro fattore decisivo è la conoscenza di lingue straniere che permette di accettare delle strutture provenienti da un'altra lingua. In certi casi il motivo è la differenziazione insufficiente dei campi semantici della parola. A seconda del modello straniero che differenzia p.es. sognare nel senso di 'immaginare in sogno' e nel senso di 'desiderare' si prende in prestito una parola straniera che sostituirà la parola originale in uno dei suoi significati. In questo modo il francese rêver è stato introdotto nel patois dei Vosgi accanto a sõže ${ }^{9}$. Non si deve però dimenticare che la causa frequente dell'introduzione delle parole straniere è il prestigio della lingua in questione. Si crede che il valore della lingua determini la posizione sociale dell'individuo. L'ultima causa rievocata è la trascuratezza dell'enunciato. Quando l'attenzione si focalizza sul tema e meno sulla lingua il transfert è frequente (Weinreich, 1953: 56-60).

\footnotetext{
${ }^{8}$ Per questa ragione il francese vomir è stato sostituito da rejeter e rendre.

${ }^{9}$ Op. cit., p. 59. Il motivo era di differenziare sul modello francese rêver e songer.
} 


\section{ERRORI INTERFERENZIALI}

In questo punto analizzeremo alcuni esempi di errori interferenziali accaduti nella produzione scritta e parlata degli studenti polacchi di filologia italiana. Gli errori sono raggruppati in tre categorie: errori fonetici, morfosintattici e lessicali.

Tra gli errori fonetici abbiamo scelto gli esempi seguenti: la parola secondo pronunciata come [segondo] che rivela l'ispirazione dal francese second [səgã] perché il francese è la lingua conosciuta meglio dal parlante. Siccome la grafia dei due lessemi è molto simile, il parlante ha la tendenza a immedesimare anche la pronuncia. Un'altra teoria spiega la sonorizzazione di $[\mathrm{k}]$ con l'assimilazione a distanza sotto l'influsso dei fonemi sonori [nd]. Un altro esempio del genere è la pronuncia della parola camion alla francese [kamjã] mentre in italiano vige la pronuncia [kàmion]. In questo caso l'errore viene spiegato con la forma originaria del lessema che è un prestito non-adattato dal francese. Similmente, essere abituati alla pronuncia francese spinge a pronunciare il lessema italiano qualità come [kalità] al posto di [kwalità] sul modello di qualité [kalite] francese.

Per quanto riguarda la morfosintassi possiamo individuare alcuni problemi che appaiono nei compiti esaminati: l'uso di articoli, di aggettivi possessivi, di pronomi ma anche del sistema verbale. Gli errori sono dovuti alle differenze tra il sistema italiano e quello francese. Tra gli errori che accadono più frequentemente vogliamo soffermarci su due casi. Il primo è la somiglianza della forma dei pronomi relativi chi, che e qui, que. A causa di questa somiglianza gli studenti creano gli enunciati di tipo: *Les personnes qu'ont beaucoup d'argent sont heureuses (Les personnes qui ont beaucoup d'argent sont heureuses) ispirato dall'italiano Le persone che hanno molti soldi sono felici oppure *Questo è l'uomo chi mi ha dato dieci euro (Questo è l'uomo che mi ha dato dieci euro) creato sull'esempio del francese C'est l'homme qui m'a donné dix euros. Un altro errore frequente è l'uso sbagliato del congiuntivo / subjonctif, spiegabile dal fatto che in italiano alcuni verbi richiedono il congiuntivo mentre i loro equivalenti francesi vengono accompagnati dall'indicativo: *Ils pensent que l'euthanasie soit immorale (Ils pensent que l'euthanasie est immorale) secondo Pensano che l'eutanasia sia immorale e *J'espère que tu sois heureux (J'espère que tu es heureux) - Spero che tu sia felice. L'uso del congiuntivo / subjonctif viene complicato da un altro fattore: in francese si impiegano solo due tipi di subjonctif mentre in italiano nell'uso comune funzionano quattro tipi del congiuntivo, il che può provocare errori di tipo: *Non pensavo che lui abbia ragione (Non pensavo che lui avesse ragione) secondo il francese Je ne pensais pas qu'il ait raison.

Nel campo del lessico è neccesario menzionare numerosi falsi amici italiano-francesi come salir - salire, anniversaire - anniversario, assez - assai, parents parenti, c'est - c'è oppure accident - accidente. Un altro problema maggiore sono gli errori di grafia tra cui i più tipici sono: *Maria est bella - Maria è bella (sotto l'influsso di Marie est belle), *Pietro et Paolo - Pietro e Paolo (Pierre et Paul), 
*Il abite - Il habite (Lui abita), *allors - alors (allora), *quatro - quattro (quatre) e*quattre - quatre (quattro), *édèle (fidèle) - fedele e *filologie - philologie (filologia).

Alcuni studenti tendono a creare delle voci inesistenti come: *viando - carne (viande), *concombre - cetriolo (concombre) e *terminazione - desinenza (terminaison). Gli esempi sopracitati sono la prova del ricorso alla lingua conosciuta meglio nel caso della mancanza di un lessema. Per colmare le lacune si impiegano i termini dell'altro sistema, il che dà spesso i risultati positivi ma si lega sempre col rischio di errori interferenziali.

\section{CONCLUSIONI}

L'analisi che abbiamo effettuato ci permette di osservare che la conoscenza precedente di una lingua straniera facilita la comprensione degli enunciati in un'altra lingua ma ne può ostacolare la produzione. In seguito, abbiamo notato che gli errori interferenziali appaiono in tutti sottosistemi della lingua: quello fonetico, morfosintattico e lessicale, anche se gli errori fonetici sono decisamente meno frequenti degli altri. La quantità di errori interferenziali italiano-francesi che abbiamo individuato conferma la teoria che la vicinanza genetica tra le lingue genera errori. Alla fine dobbiamo stabilire che il corpus rilevato costituisce solo un campione e il suo ampliamento darà sicuramente dei risultati più differenziati. Tuttavia, la consapevolezza di commettere errori è il primo passo per eliminarli.

\section{BIBLIOGRAFIA}

Bettoni C. (1999), «Il bilinguismo ", Italiano e oltre, XIV, p. 230-238.

Czochralski J.A. (1979), « O interferencji językowej », in: Polska myśl glottodydaktyczna 1945-1975: wybór artykułów z zakresu glottodydaktyki ogólnej, dir. F. Grucza, Warszawa : PWN.

Czochralski J.A. (1979), “Wybrane zagadnienia interferencji strukturalnej », in: Polska myśl glottodydaktyczna 1945-1975: wybór artykułów z zakresu glottodydaktyki ogólnej, dir. F. Grucza, Warszawa : PWN.

George H.V. (1972), Common errors in language learning. Insights from English, Rowley : Newbury House Publishers.

Grochowski L. (1979), « Interferencja językowa z psychologicznego i lingwistycznego punktu widzenia a proces nauczania języka rosyjskiego », in: Polska myśl glottodydaktyczna 1945-1975: wybór artykutów z zakresu glottodydaktyki ogólnej, dir. F. Grucza, Warszawa : PWN.

Grucza F. (1981), «Glottodydaktyczne implikacje bilingwizmu » in: Bilingwizm a glottodydaktyka. Materiaty z V Sympozjum zorganizowanego przez Instytut Lingwistyki Stosowanej UW, Biatowie¿a 26-28 maja 1977, Warszawa: Wydawnictwo UW.

Gusmani R. (1981), Saggi sull'interferenza linguistica, Firenze : Casa Editrice Le Lettere.

Komorowska H. (1980), Nauczanie gramatyki języka obcego a interferencja, Warszawa: WSiP. 
Lipińska E. (2003), Język ojczysty, język obcy, język drugi...Wstęp do badań dwujęzyczności, Kraków : Wydawnictwo UJ.

Łuczyński E., Maćkiewicz J. (1999), Językoznawstwo ogólne. Wybrane zagadnienia, Gdańsk : Wydawnictwo Uniwersytetu Gdańskiego.

Odlin T. (1989), Language Transfer. Cross-linguistic influence in language learning, Cambridge: Cambridge University Press.

Prejbisz A. (1964), « Zagadnienie interferencji w nauczaniu języka obcego », Języki Obce w Szkole, 4/1964, p. 213-225.

Rivers Wilga M. (1972), Speaking in Many Tongues: Essays in Foreign-Language Teaching, Rowley : Newbury House Publishers.

Rudolf K.F. (2003), Fatszywi przyjaciele - false friends. Stownik angielsko-polski, polsko-angielski wyrazów zdradliwych, Kraków: Wydawnictwo Literackie.

Szulc A. (1979), « Podstawy ogólnej teorii interferencji », in: Polska myśl glottodydaktyczna 1945 1975: wybór artykutów z zakresu glottodydaktyki ogólnej, dir. F. Grucza, Warszawa : PWN.

Weinreich U. (1953), Languages in contact. Findings and problems, the Hague : Mouton Publishers.

Wilczyńska W., Rabiller B. (1995), Stownik pułapek jezykowych polsko-francuski = Dictionnaire des mots-piẻges polonais-français, Warszawa : Wiedza Powszechna.

Woźniakowski W. (1981), «Glottodydaktyczna relewancja badań nad bilingwizmem », in: Bilingwizm a Glottodydaktyka. Materiahy z V Sympozjum zorganizowanego przez Instytut Lingwistyki Stosowanej UW, Biatowieza 26-28 maja 1977, Warszawa : Wydawnictwa Uniwersytetu Warszawskiego.

Wróblewska-Pawlak K. (2003), « Co to znaczy być dwujęzycznym? Definicje teoretyczne a społeczne reprezentacje dwujęzyczności ", in: Problemy lingwistyki i nauczania języków obcych, dir. H. Miatliuk, K. Bogacki et H. Komorowska, Bialystok : Uniwersytet w Białymstoku. 